\title{
Isolation and Identification of Cellulose Degrading Bacteria from Mangrove Soil of Mahanadi River Delta and Their Cellulase Production Ability
}

\author{
B.C.Behera ${ }^{1}$, S.Parida ${ }^{2}$, S.K.Dutta ${ }^{3}$, H.N.Thatoi ${ }^{4, *}$ \\ ${ }^{1}$ Department of Biotechnology, North Orissa University, Baripada, India \\ ${ }^{2}$ Department of Biotechnology, MITS School of Biotechnology, Bhubaneswar, India \\ ${ }^{3}$ Centre for Ecological Sciences, Indian Institute of Science, Bangalore, India \\ ${ }^{4}$ Department of Biotechnology, College of Engineering and Technology (BPUT), Bhubaneswar, India \\ *Corresponding author: hn_thatoi@rediffmail.com
}

Received October 19, 2013; Revised November 27, 2013; Accepted February 07, 2014

\begin{abstract}
This study was conducted to isolate cellulose degrading bacteria from mangrove soil of Mahanadi river delta, Odisha, India and also to evaluate their cellulase production ability. Results showed that in total fifteen cellulose degrading bacteria were isolated based on their halo zone formation on congored agar medium. Their maximum carboxymethylcellulose hydrolysis capacities (HC value) were ranged from 1.18 to $2.5 \mathrm{~cm}$. A CMCase test of these fifteen isolates showed that their enzyme activity ranged from 2.471 to $98.253 \mathrm{U} / \mathrm{ml} / \mathrm{min}$. From the morphological, aand biochemical characterisation, the isolates were identified as Micrococcus spp., Bacillus spp., Pseudomonas spp., Xanthomonas spp. and Brucella spp.
\end{abstract}

Keywords: mangrove, cellulose degrading bacteria, cellulase, biochemical characterisation

Cite This Article: B.C.Behera, S.Parida, S.K.Dutta, and H.N.Thatoi, "Isolation and Identification of Cellulose Degrading Bacteria from Mangrove Soil of Mahanadi River Delta and Their Cellulase Production Ability.” American Journal of Microbiological Research, vol. 2, no. 1 (2014): 41-46. doi: 10.12691/ajmr-2-1-6.

\section{Introduction}

Mangroves are highly productive marine ecosystem where bacteria actively take part in bio-mineralization and biotransformation of minerals [1]. The distribution of microbial activities in estuarine systems is clearly complex and variable. Leaves and wood provided by mangrove plants to the soil are degraded primarily by large variety of microbes which actively participate in the heterotrophic food chain [2]. Major products of general recycling of organic matter are detritus which is rich in enzymes and proteins and contains large micro-bial population [3]. Thus mangrove-derived detritus constitutes a large reservoir of carbon and energy potentially available to the estuarine food web and Bacteria are the major participants in the carbon, sulfur, nitrogen and phosphorous cycles in mangrove forest [4]. Roughly $30 \%$ to $50 \%$ of the organic matters in mangrove leaves are leachable water-soluble compounds such as tannins and sugars [5], the remaining fraction of the organic matter presumably consists of plant structural polymers commonly referred to as cellulosic substance. Cellulose is a linear polysaccharide which is constructed from monomer of glucose bound together with 1-4 glucosidal linkage [6]. The transfer of carbon and energy from mangrove detritus to animal consumers appears to occur via grazing of easily digestible and highly nutritive microbial biomass resulting from bacterial and fungal transformation of the lignocellulosic detritus. Thus most of the bacteria and fungi degrade this cellulosic material by producing enzyme called cellulase. Cellulase is the enzyme that hydrolyse the $\beta$-1, 4-glycosidic bonds in the polymer to release glucose units [7]. Cellulases are among the industrially important hydrolytic enzymes and are of great significance in present day biotechnology. Cellulases are widely used in the food, feed, textile and pulp industries [8]. The bioconversion of cellulosic materials is now a subject of intensive research as a contribution to the development of large scale conversion process beneficial to mankind [9].

Considering this huge significance of cellulase which contribute $8 \%$ of the world wide industrial enzyme demand and the demand is expected to increase by $100 \%$ within 2014 [10], surprisingly little is known about the bacterial and fungal cellulase in mangrove swamps and adjacent coastal environments. Keeping the above in vision, the present study aimed to isolate and characterize bacterial isolates with high cellulase-producing ability from mangrove soil of Mahanadi river delta, Odisha, India.

\section{Materials and Methods}

\subsection{Isolation of Cellulose Degrading Bacteria}

The soil samples were collected from different location of mangrove forest such as Jumbo, Kharnasi, Triveni, 
Nuagada, Atharabanki and Mangrove forest at Indian Farmer fertilisers Corporation (IFFCO). After collection, the samples were stored at $4^{\circ} \mathrm{C}$ in sterile containers. For each soil sample, several sub-samples were taken, homogenized in sterile milli Q water containing $0.85 \%$ $\mathrm{NaCl}(\mathrm{w} / \mathrm{v})$ and serially diluted and pour plate and spread plate techniques were done using Carboxymethylcellulose (CMC) agar media [11]. The plates were incubated at $37^{\circ} \mathrm{C}$ for $24-48 \mathrm{~h}$. To visualize the hydrolysis zone, the plates were flooded with $0.1 \%$ Congo red solution and washed with $1 \mathrm{M} \mathrm{NaCl}$ [11]. The formation of a clear zone of hydrolysis indicated cellulose degradation. The ratio of the clear zone diameter to colony diameter was measured in order to select for the highest cellulase activity producer. The largest ratio was assumed to contain the highest activity $[12,13]$.

\subsection{Cellulase Activity Test of the Isolates}

For the quantitative estimation of cellulase, the bacterial isolates were grown in Carboxymethyl cellulose broth medium contain (g/l): Carboxymethylcellulose (CMC), 10; Tryptone, 2; $\mathrm{KH}_{2} \mathrm{PO}_{4}$, 4; $\mathrm{Na}_{2} \mathrm{HPO}_{4}$, 4; $\mathrm{MgSO}_{4} .7 \mathrm{H}_{2} \mathrm{O}, 0.2$; $\mathrm{CaCl}_{2} .2 \mathrm{H}_{2} \mathrm{O}, 0.001$; $\mathrm{FeSO}_{4} .7 \mathrm{H}_{2} \mathrm{O}, 0.004$; Agar, 15 and $\mathrm{pH}$ adjusted to 7 [14]. CMCase activity was assayed by using methode described by [15]. The activity was estimated using $1 \%$ solution of carboxymethyl cellulose (CMC) in $0.05 \mathrm{M}$ citrate buffer ( $\mathrm{pH} 4.8)$ as substrate. The reaction mixture was contained $0.5 \mathrm{ml}$ of substrate solution and 0.5 $\mathrm{ml}$ of suitably diluted enzyme solution. The reaction was carried out at $50^{\circ} \mathrm{C}$ for 30 minutes and the reaction was stopped by adding $3 \mathrm{ml}$ of DNS solution to the reaction mixture. The reaction mixture was then boiled at $100^{\circ} \mathrm{C}$ for 5 minute. O.D. was taken with systronics 119 spectrophotometer at $540 \mathrm{~nm}$. One unit of CMCase activity was expressed as $1 \mu$ mol of glucose liberated per $\mathrm{ml}$ of enzyme per minute. The values obtained are compared with Glucose standard curve.

\subsection{Identification of the Isolates}

The bacterial isolates were presumptively identified by means of morphological examination and some biochemical characterisation. The parameters investigated included colony characteristics, shape, size, spore, motility, Gram's reaction, catalase production, urease production, Voges-Proskauer (V-P) reaction, Indole production, Nitrate reduction, citrate utilization, carbohydrate metabolism (acid-gas production), starch hydrolysis, Tributyrin (or vegetable oil) hydrolysis, Tween-80 hydrolysis, Cholesterol hydrolysis, gelatin hydrolysis, Casein hydrolysis, Growth at different $\mathrm{pH}$ and Temperature, Pectin hydrolysis and chitin hydrolysis test were carried out following the standard methods described in Bergey's Manual of Determinative Bacteria [16]. Response of the organism to different antibiotics was tested on NA medium. NA plates were surface seeded with concentrated bacterial suspension. Different antibiotic discs with effective concentrations were placed over the plates. Inhibition of growth depicted by a clear zone formation around the discs indicated sensitive reaction otherwise the organism was resistant to the antibiotic. Diameter of the inhibition zone was measured with an antibiotic zone scale. Ratio of the inhibition zone and disc area produced the activity level of the antibiotics.

\subsection{Statistical Analysis}

Statistical analysis was performed by SPSS, version 10 for windows (SPSS Inc; Chicago, IL, USA).

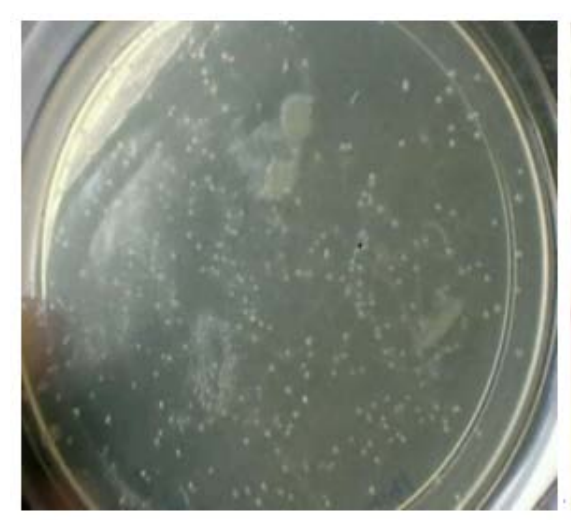

a

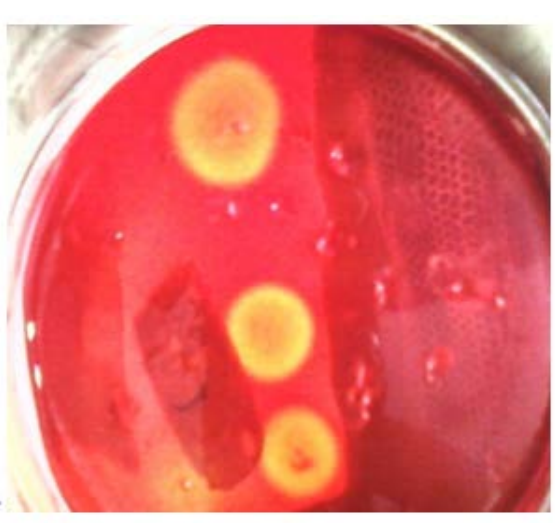

b

Figure 1. (a) CMC agar plate before staining with Congored (b) CMC agar plate after staining with congored and $\mathrm{NaCl}$

\section{Results and Discussion}

Cellulose degrading bacteria were isolated from mangrove soils of Mahanadi delta using CMC agar medium (Figure 1. a). 15 morphologically distinct bacterial isolates which were giving halo zones in CMC agar medium were selected and named as Cellulose degrading bacteria, CDB1-15 (Figure 1. b). In the present study, less number of cellulose degrading bacteria were observed from mangrove soil of Mahanadi delta in comparison to the finding of Ramanathan et al., [17], who observed comparatively large number of cellulose degrading bacteria from Sundarban mangroves of West Bengal, India. The clearing zone size and colony diameter of these isolates were measured when incubated aerobically at $37^{\circ} \mathrm{C}$; the result showed that maximum clearing zone ranged from 0.9 and $2.1 \mathrm{~cm}$ and the average $\mathrm{HC}$ value, i.e. ratio of zone size to colony diameter ranged from 1.18 to $2.5 \mathrm{~cm}$, demonstrating that all the isolates have the ability to degrade the carboxymethyl cellulose and indicating high ability of $\mathrm{Cx}$ cellulase production (Table 1). Distinct Cx cellulase production was detected by bacterial isolate CDB-5 as evidenced by its maximum HC value of 2.5. This finding is very similar to the 
findings of Hatami et al. [18], who also observed the ratio of the zone diameter to colony diameter ratio 0.4 to 2.1 . Lu et al. [19], observed maximum clearing zone ranged between 2.5 to $6.4 \mathrm{~cm}$ with maximum HC value of 4.85-
$13.11 \mathrm{~cm}$. But the observed HC value is lower than the value observed by Gupta et al. [20], who observed maximum HC value of 9 to $9.8 \mathrm{~cm}$.

Table 1. Evaluation of cellulase activity of some cellulose degrading bacteria in CMC agar plate through halo zone formation

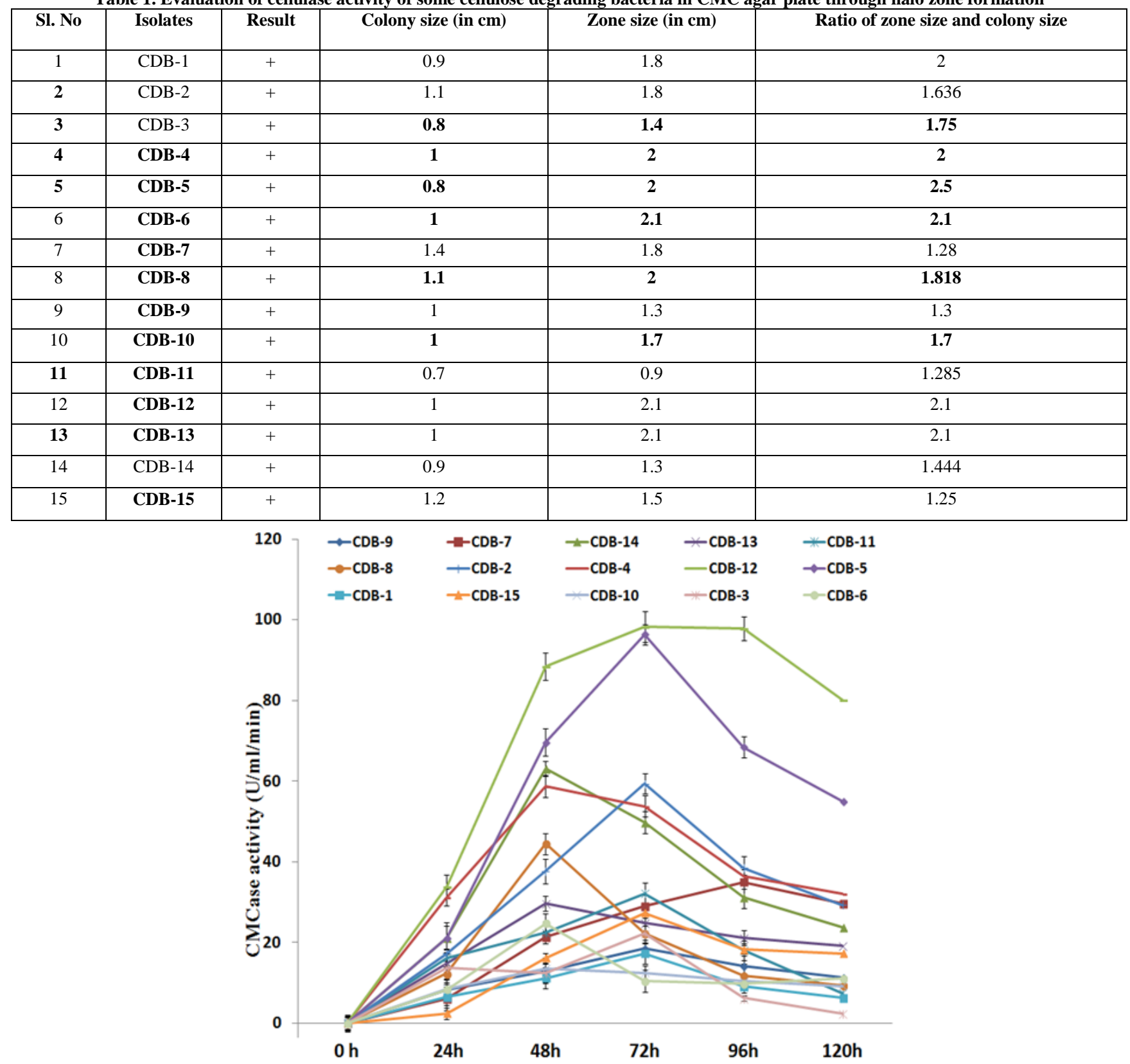

Incubation period (h)

Figure 2. Cellulase production ability of the fifteen cellulose degrading bacterial isolates from mangrove soil of Mahanadi river delta, Odisha, India

\subsection{Measurement of Cellulase Production}

Experiments were carried out to determine the cellulase production ability of 15 bacterial isolates (CDB-1, CDB-2, CDB-3, CDB-4, CDB-5, CDB-6, CDB-7, CDB-8, CDB-9, CDB-10, CDB-11, CDB-12, CDB-13, CDB-14 and CDB$15)$. The cellulase production ability of the bacterial isolates in the production medium were in the following order, CDB-10 (13.591 U/ml/min) < CDB-1 (17.321 $\mathrm{U} / \mathrm{ml} / \mathrm{min})<$ CDB-9 $(18.53 \mathrm{U} / \mathrm{ml} / \mathrm{min})<$ CDB-3 $(22.24$ $\mathrm{U} / \mathrm{ml} / \mathrm{min})<$ CDB-6 (24.71 U/ml/min) $<$ CDB-15 (27.298 $\mathrm{U} / \mathrm{ml} / \mathrm{min})<$ CDB-13 (29.653 U/ml $/ \mathrm{min})<$ CDB-11 (32.124 U/ml/min) < CDB-7 (34.971 U/ml/min) < CDB-8
(44.48 U/ml/min) < CDB-4 (58.701 U/ml/min) < CDB-2 $(59.307 \mathrm{U} / \mathrm{ml} / \mathrm{min})<$ CDB-14 $(63.013 \mathrm{U} / \mathrm{ml} / \mathrm{min})<$ CDB5 (96.374 U/ml/min) < CDB-12 (98.253 U/ml/min). Their cellulase production efficiency ranged between 2.471 to 98.253 U/ml/min (Figure 2). Among these two bacterial isolate CDB-12 showed maximum cellulase production ability of $98.253 \mathrm{U} / \mathrm{ml} / \mathrm{min}$ followed by CDB-5 (96.374 $\mathrm{U} / \mathrm{ml} / \mathrm{min}$ ). Our finding is higher than the finding of Tabo and Monsalud [21] who observed $56.60 \mathrm{U} / \mathrm{ml}$, (Bacillus cereus), $66.50 \mathrm{U} / \mathrm{ml}$ (Bacillus licheniformis), $50.33 \mathrm{U} / \mathrm{ml}$, (Bacillus spp.), $51.04 \mathrm{U} / \mathrm{ml}$, (Bacillus pumilus), 48.70 $\mathrm{U} / \mathrm{ml}$, (Bacillus pumilus) of cellulase activity from philipines mangrove soil. Recently it has also been 
reported the cellulase production by Bacillus cereus strain

is nearly similar to the cellulase production by the up to $102 \mathrm{U} / \mathrm{ml}$ at 48 hour of incubation [22]. This finding

bacterial isolate CDB-12 and CDB-5 in the present study.

\begin{tabular}{|c|c|c|c|c|c|c|c|c|c|c|c|c|c|c|c|}
\hline $\begin{array}{l}\text { Characte } \\
\text { rs }\end{array}$ & $\begin{array}{l}\text { CDB } \\
1\end{array}$ & $\begin{array}{l}\text { CDB } \\
2\end{array}$ & $\begin{array}{l}\text { CDB } \\
3\end{array}$ & $\begin{array}{l}\text { CDB } \\
4\end{array}$ & $\begin{array}{l}\text { CDB } \\
5\end{array}$ & $\begin{array}{l}\text { CDB } \\
6\end{array}$ & $\begin{array}{l}\text { CDB } \\
7\end{array}$ & $\begin{array}{l}\text { CDB } \\
8\end{array}$ & $\begin{array}{l}\text { CDB } \\
9\end{array}$ & $\begin{array}{l}\text { CDB1 } \\
0\end{array}$ & $\begin{array}{l}\text { CDB1 } \\
1\end{array}$ & $\begin{array}{l}\text { CDB1 } \\
2\end{array}$ & $\begin{array}{l}\text { CDB1 } \\
3\end{array}$ & $\begin{array}{l}\text { CDB1 } \\
4\end{array}$ & $\begin{array}{l}\text { CDB1 } \\
5\end{array}$ \\
\hline Shape & Rod & Rod & Rod & cocci & Rod & cocci & Rod & Rod & Rod & Rod & Rod & Rod & Rod & Rod & cocci \\
\hline $\begin{array}{l}\text { Cell } \\
\text { diameter } \\
(\mu \mathrm{m})\end{array}$ & $\begin{array}{l}0.8- \\
0.9\end{array}$ & $\begin{array}{l}0.7- \\
0.8\end{array}$ & $\begin{array}{l}0.8- \\
0.1\end{array}$ & $\begin{array}{l}0.8- \\
2.0\end{array}$ & $\begin{array}{l}0.5- \\
0.7\end{array}$ & $\begin{array}{l}0.9- \\
2.1\end{array}$ & $\begin{array}{l}0.9- \\
0.1\end{array}$ & $\begin{array}{l}0.5- \\
0.7\end{array}$ & $\begin{array}{l}1.0- \\
1.05\end{array}$ & $\begin{array}{l}0.7- \\
0.8\end{array}$ & $\begin{array}{l}0.9- \\
1.0\end{array}$ & $\begin{array}{l}1.55- \\
1.98\end{array}$ & $\begin{array}{l}0.9- \\
1.0\end{array}$ & $\begin{array}{l}1.55- \\
1.98\end{array}$ & $\begin{array}{l}1.0- \\
2.1\end{array}$ \\
\hline Spore & + & + & - & - & - & - & - & - & + & - & - & + & - & + & - \\
\hline $\begin{array}{l}\text { Motility } \\
\text { test }\end{array}$ & + & + & + & - & - & - & + & - & + & + & + & - & + & - & - \\
\hline $\begin{array}{l}\text { Aerobic } \\
\text { growth }\end{array}$ & + & + & + & + & + & + & + & + & + & + & + & + & + & + & + \\
\hline $\begin{array}{l}\text { Urease } \\
\text { test }\end{array}$ & - & - & - & + & + & + & - & + & - & - & + & + & + & + & + \\
\hline $\begin{array}{l}\text { Gram } \\
\text { stain }\end{array}$ & + & + & - & + & - & + & - & - & + & - & - & + & - & + & + \\
\hline PHB & ND & ND & + & - & ND & - & + & ND & - & - & - & - & - & - & - \\
\hline $\begin{array}{l}\text { Catalase } \\
\text { test }\end{array}$ & + & + & + & + & + & + & + & + & + & + & + & + & + & + & + \\
\hline MR & - & - & - & - & - & - & - & - & + & - & + & - & + & - & - \\
\hline VP & - & - & - & - & - & - & - & - & + & - & - & - & - & - & - \\
\hline $\begin{array}{l}\text { Citrate } \\
\text { utilisation }\end{array}$ & + & - & - & + & + & + & - & + & + & + & + & + & + & + & + \\
\hline \multicolumn{16}{|l|}{ Protease: } \\
\hline $\begin{array}{l}\text { Gelatin } \\
\text { hydrolysis }\end{array}$ & + & + & + & - & + & - & - & + & + & - & - & - & - & - & - \\
\hline $\begin{array}{l}\text { Casein } \\
\text { hydrolysis }\end{array}$ & + & + & + & - & + & - & + & + & + & - & - & - & - & - & - \\
\hline \multicolumn{16}{|l|}{ Lipase: } \\
\hline $\begin{array}{l}\text { Tributyrin } \\
\text { hydrolysis }\end{array}$ & ND & ND & ND & & - & & ND & - & - & + & - & - & - & - & \\
\hline $\begin{array}{l}\text { Tween } 80 \\
\text { hydrolysis }\end{array}$ & ND & ND & ND & - & + & - & ND & + & + & + & - & - & - & - & - \\
\hline $\begin{array}{l}\text { Lecithinas } \\
\text { e egg yolk }\end{array}$ & - & - & - & - & - & - & - & - & - & - & + & + & + & + & - \\
\hline $\begin{array}{l}\text { Chitinase } \\
\text { productio } \\
\text { n }\end{array}$ & - & - & - & & - & & - & - & + & - & - & - & - & - & \\
\hline $\begin{array}{l}\text { Argine } \\
\text { dihydrola } \\
\text { se }\end{array}$ & - & - & - & - & - & - & - & - & + & - & + & - & + & - & - \\
\hline $\begin{array}{l}\text { Strach } \\
\text { hydrolysis }\end{array}$ & + & + & + & - & + & - & - & + & + & + & + & - & + & - & - \\
\hline $\begin{array}{l}\text { Oxidase } \\
\text { productio } \\
\mathrm{n}\end{array}$ & - & + & - & + & + & + & - & + & + & + & - & + & - & + & + \\
\hline $\begin{array}{l}\text { Nitrate } \\
\text { reduction }\end{array}$ & + & - & - & + & + & + & - & + & + & + & - & + & - & + & + \\
\hline \multicolumn{16}{|l|}{$\begin{array}{l}\text { Acid } \\
\text { from: }\end{array}$} \\
\hline Glucose & ND & ND & + & - & - & - & + & - & + & + & + & - & + & - & - \\
\hline Fructose & ND & ND & + & - & - & - & + & - & + & + & + & - & + & - & - \\
\hline Mannose & ND & ND & + & - & - & - & + & - & + & + & + & - & + & - & - \\
\hline $\begin{array}{l}\text { Gas } \\
\text { productio } \\
\text { n }\end{array}$ & - & - & - & - & - & - & - & - & - & - & - & - & - & - & - \\
\hline Glucose & ND & ND & - & - & - & & - & - & - & + & - & - & - & - & \\
\hline Indole & - & - & - & - & - & & - & - & - & - & - & - & - & - & \\
\hline $\begin{array}{l}\text { Growth } \\
\text { pH 5-7 }\end{array}$ & + & + & + & + & + & + & + & + & + & + & + & + & + & + & + \\
\hline $\begin{array}{l}\text { Growth } \\
40^{\circ} \mathrm{C}\end{array}$ & + & + & + & + & + & + & + & + & + & + & + & + & + & + & + \\
\hline
\end{tabular}




\subsection{Identification of the Isolates}

These fifteen selected bacterial isolates were subjected to various morphological and Biochemical characterisation (Table 2) and antibiotic sensitivity test (Table 3) with a view to identify them. The colony characteristics of the isolates were found variable. The isolates were undulate, convex, and circular having gummy and sticky consistency. The size of the vegetative cell of the isolates was 0.8 to $2.1 \mu \mathrm{m}$. Microscopic observation of the isolates revealed that most of them are rod shaped and motile. The isolates were found variable towards Gram's stain. All the isolates were sensitive to penicillin G, polymyxin B, Erythromycin, Gentamycin and Ciprofloxacin and resistant to Nystatin. Except CDB7 all the isolates were sensitive to norfloxacin and bacitracin. Similarly except CDB-7 and CDB-13 all other isolates are sensitive to chloromphenicol. Only CDB-8, CDB-12 and CDB-13 were only resistant to Tetracyclin.
On the basis of their morphological characterisation, antibiotic sensitivity test and biochemical characterisation these isolates were identified as Bacillus brevis (CDB1), Bacillus alcalophilus (CDB-2) Xanthomonas spp. (CDB3), Micrococcus spp (CDB-4), Brucella spp (CDB5), Micrococcus spp (CDB-6), Xanthomonas spp (CDB7), Brucella spp (CDB8), Bacillus subtilis (CDB-09), Pseudomonas stutzeri (CDB10), Pseudomonas spp (CDB11), Bacillus spp (CDB-12), Pseudomonas spp (CDB13), Bacillus spp (CDB-14) and Micrococcusspp (CDB-15). Our finding is similar to the findings of Tabo and Monsalud [21] who also reported occurrence of Bacillus cereus, Bacillus licheniformis, Bacillus spp, and Bacillus pumilus from philipines mangrove soil. Thatoi et al. [23], also reported the cellulytic activity of Pseudomonas spp, Bacillus spp, Bacillus polymyxa, and Bacillus brevis from mangrove soil of Bhitarakanika, Odisha.

Table 3. Antibiotic sensitivity test of fifteen cellulose degrading bacterial isolates

\begin{tabular}{|c|c|c|c|c|c|c|c|c|c|c|c|c|c|c|c|c|c|c|c|c|c|c|c|c|c|c|c|c|c|c|}
\hline \multirow{2}{*}{$\begin{array}{l}\text { Antibiot } \\
\text { ics }\end{array}$} & \multicolumn{2}{|c|}{$\begin{array}{l}\text { CDB } \\
1\end{array}$} & \multicolumn{2}{|c|}{$\begin{array}{l}\text { CDB } \\
2\end{array}$} & \multicolumn{2}{|c|}{$\begin{array}{l}\text { CDB } \\
3\end{array}$} & \multicolumn{2}{|c|}{$\begin{array}{l}\text { CDB } \\
4\end{array}$} & \multicolumn{2}{|c|}{$\begin{array}{l}\text { CDB } \\
5\end{array}$} & \multicolumn{2}{|c|}{$\begin{array}{l}\text { CDB } \\
6\end{array}$} & \multicolumn{2}{|c|}{$\begin{array}{l}\text { CDB } \\
7\end{array}$} & \multicolumn{2}{|c|}{$\begin{array}{l}\text { CDB } \\
8\end{array}$} & \multicolumn{2}{|c|}{$\begin{array}{l}\text { CDB } \\
9\end{array}$} & \multicolumn{2}{|c|}{$\begin{array}{l}\text { CDB } \\
10\end{array}$} & \multicolumn{2}{|c|}{$\begin{array}{l}\text { CDB } \\
11\end{array}$} & \multicolumn{2}{|c|}{$\begin{array}{l}\text { CDB } \\
12\end{array}$} & \multicolumn{2}{|c|}{$\begin{array}{l}\text { CDB } \\
13\end{array}$} & \multicolumn{2}{|c|}{$\begin{array}{l}\text { CDB } \\
14\end{array}$} & \multicolumn{2}{|c|}{$\begin{array}{l}\text { CDB } \\
15\end{array}$} \\
\hline & $\begin{array}{l}\mathrm{S} \\
1 \\
\mathrm{R} \\
\end{array}$ & C & $\begin{array}{l}\mathrm{S} \\
1 \\
\mathrm{R} \\
\end{array}$ & C & $\begin{array}{l}\mathrm{S} \\
1 \\
\mathrm{R} \\
\end{array}$ & C & $\begin{array}{l}\mathrm{S} \\
1 \\
\mathrm{R} \\
\end{array}$ & C & $\begin{array}{l}\mathrm{S} \\
1 \\
\mathrm{R} \\
\end{array}$ & C & $\begin{array}{l}\mathrm{S} \\
1 \\
\mathrm{R} \\
\end{array}$ & C & $\begin{array}{l}\mathrm{S} \\
1 \\
\mathrm{R} \\
\end{array}$ & C & $\begin{array}{l}\mathrm{S} \\
/ \\
\mathrm{R} \\
\end{array}$ & $C$ & \begin{tabular}{l|l}
$\mathrm{S}$ \\
1 \\
$\mathrm{R}$ \\
\end{tabular} & $\mathrm{C}$ & \begin{tabular}{|l|}
$\mathrm{S}$ \\
1 \\
$\mathrm{R}$ \\
\end{tabular} & C & \begin{tabular}{l|l}
$\mathrm{S}$ \\
$/$ \\
$\mathrm{R}$
\end{tabular} & C & \begin{tabular}{l|l}
$\mathrm{S}$ \\
1 \\
$\mathrm{R}$
\end{tabular} & C & \begin{tabular}{l|}
$\mathrm{S}$ \\
1 \\
$\mathrm{R}$ \\
\end{tabular} & $\mathrm{C}$ & \begin{tabular}{l|}
$\mathrm{S}$ \\
1 \\
$\mathrm{R}$ \\
\end{tabular} & C & \begin{tabular}{|l|}
$\mathrm{S}$ \\
1 \\
$\mathrm{R}$ \\
\end{tabular} & $\mathrm{C}$ \\
\hline $\begin{array}{l}\text { Nystain } \\
(10 \mu \mathrm{g})\end{array}$ & $\mathrm{R}$ & - & $\mathrm{R}$ & - & $\mathrm{R}$ & - & $\mathrm{R}$ & - & $\mathrm{R}$ & - & $\mathrm{R}$ & - & $\mathrm{R}$ & - & $\mathrm{R}$ & - & $\mathrm{R}$ & - & $\mathrm{R}$ & - & $\mathrm{R}$ & & $\mathrm{R}$ & - & $\mathrm{R}$ & & $\mathrm{R}$ & & & \\
\hline $\begin{array}{l}\text { Penicilli } \\
\text { n G (10 } \\
\mu \mathrm{g})\end{array}$ & S & $\begin{array}{l}1 \\
6\end{array}$ & $S$ & $\begin{array}{l}3 \\
6\end{array}$ & $S$ & 2 & S & $\begin{array}{l}3 \\
6\end{array}$ & S & $\begin{array}{l}1 \\
6\end{array}$ & S & $\begin{array}{l}1 \\
9\end{array}$ & S & $\begin{array}{l}2 \\
7\end{array}$ & S & $\begin{array}{l}2 \\
9\end{array}$ & $S$ & $\begin{array}{l}2 \\
1\end{array}$ & $S$ & $\begin{array}{l}2 \\
1\end{array}$ & $\mathrm{~S}$ & $\begin{array}{l}2 \\
3\end{array}$ & $S$ & $\begin{array}{l}2 \\
2\end{array}$ & $S$ & $\begin{array}{l}2 \\
4\end{array}$ & $S$ & $\begin{array}{l}2 \\
1\end{array}$ & $S$ & $\begin{array}{l}1 \\
9\end{array}$ \\
\hline $\begin{array}{l}\text { Polymy } \\
\text { xin B } \\
(300 \\
\mu \mathrm{g})\end{array}$ & S & $\begin{array}{l}1 \\
3\end{array}$ & $S$ & $\begin{array}{l}1 \\
5\end{array}$ & $S$ & $\begin{array}{l}1 \\
6\end{array}$ & S & $\begin{array}{l}1 \\
5\end{array}$ & S & $\begin{array}{l}1 \\
3\end{array}$ & $S$ & $\begin{array}{l}1 \\
3\end{array}$ & S & 9 & $S$ & $\begin{array}{l}1 \\
1\end{array}$ & $S$ & $\begin{array}{l}1 \\
6\end{array}$ & $S$ & $\begin{array}{l}1 \\
5\end{array}$ & S & $\begin{array}{l}1 \\
4\end{array}$ & $S$ & $\begin{array}{l}1 \\
6\end{array}$ & $S$ & $\begin{array}{l}1 \\
5\end{array}$ & S & $\begin{array}{l}1 \\
5\end{array}$ & S & $\begin{array}{l}1 \\
3\end{array}$ \\
\hline $\begin{array}{l}\text { Norflox } \\
\text { acin }(10 \\
\mu \mathrm{g})\end{array}$ & $S$ & $\begin{array}{l}2 \\
5\end{array}$ & S & $\begin{array}{l}1 \\
8\end{array}$ & S & 1 & S & $\begin{array}{l}1 \\
8\end{array}$ & S & $\begin{array}{l}2 \\
5\end{array}$ & S & $\begin{array}{l}2 \\
1\end{array}$ & $\mathrm{R}$ & - & S & $\begin{array}{l}2 \\
9\end{array}$ & S & $\begin{array}{l}1 \\
8\end{array}$ & S & $\begin{array}{l}2 \\
4\end{array}$ & S & $\begin{array}{l}2 \\
6\end{array}$ & S & $\begin{array}{l}2 \\
4\end{array}$ & $\mathrm{~S}$ & $\begin{array}{l}2 \\
5\end{array}$ & $\mathrm{~S}$ & $\begin{array}{l}2 \\
4\end{array}$ & S & $\begin{array}{l}2 \\
1\end{array}$ \\
\hline \begin{tabular}{l}
\multicolumn{2}{c}{ Bacitrac } \\
in $\quad(10$ \\
$\mu \mathrm{g})$
\end{tabular} & S & $\begin{array}{l}1 \\
1\end{array}$ & S & $\begin{array}{l}3 \\
2\end{array}$ & S & 1 & S & $\begin{array}{l}3 \\
2\end{array}$ & S & $\begin{array}{l}1 \\
1\end{array}$ & S & $\begin{array}{l}1 \\
9\end{array}$ & $\mathrm{R}$ & - & S & $\begin{array}{l}1 \\
9\end{array}$ & $\mathrm{~S}$ & $\begin{array}{l}1 \\
0\end{array}$ & S & $\begin{array}{l}1 \\
7\end{array}$ & S & $\begin{array}{l}1 \\
5\end{array}$ & $S$ & $\begin{array}{l}1 \\
9\end{array}$ & $\mathrm{~S}$ & $\begin{array}{l}2 \\
2\end{array}$ & $S$ & $\begin{array}{l}1 \\
7\end{array}$ & S & $\begin{array}{l}1 \\
9\end{array}$ \\
\hline $\begin{array}{l}\text { Chloro } \\
\text { mpheni } \\
\text { col (30 } \\
\mu \mathrm{g})\end{array}$ & $S$ & $\begin{array}{l}3 \\
5\end{array}$ & S & $\begin{array}{l}2 \\
1\end{array}$ & S & 2 & S & $\begin{array}{l}2 \\
1\end{array}$ & S & $\begin{array}{l}3 \\
5\end{array}$ & S & $\begin{array}{l}1 \\
3\end{array}$ & $\mathrm{R}$ & - & S & $\begin{array}{l}1 \\
2\end{array}$ & S & $\begin{array}{l}2 \\
4\end{array}$ & S & $\begin{array}{l}1 \\
5\end{array}$ & S & $\begin{array}{l}1 \\
4\end{array}$ & S & $\begin{array}{l}1 \\
6\end{array}$ & $\mathrm{R}$ & - & S & $\begin{array}{l}1 \\
5\end{array}$ & S & $\begin{array}{l}1 \\
3\end{array}$ \\
\hline $\begin{array}{l}\text { Erythro } \\
\text { mycin } \\
(15 \mu \mathrm{g})\end{array}$ & S & 6 & S & $\begin{array}{l}1 \\
4\end{array}$ & S & $\begin{array}{l}1 \\
4\end{array}$ & S & $\begin{array}{l}1 \\
4\end{array}$ & S & 6 & S & 9 & S & $\begin{array}{l}1 \\
2\end{array}$ & S & $\begin{array}{l}1 \\
3\end{array}$ & S & $\begin{array}{l}1 \\
4\end{array}$ & S & $\begin{array}{l}1 \\
3\end{array}$ & $\mathrm{~S}$ & $\begin{array}{l}1 \\
1\end{array}$ & $\mathrm{~S}$ & $\begin{array}{l}1 \\
2\end{array}$ & $\mathrm{~S}$ & $\begin{array}{l}1 \\
3\end{array}$ & S & $\begin{array}{l}1 \\
3\end{array}$ & S & 9 \\
\hline $\begin{array}{l}\text { Gentam } \\
\text { ycin }(10 \\
\mu \mathrm{g})\end{array}$ & S & $\begin{array}{l}3 \\
9\end{array}$ & S & $\begin{array}{l}3 \\
9\end{array}$ & S & $\begin{array}{l}2 \\
9\end{array}$ & S & $\begin{array}{l}3 \\
9\end{array}$ & S & $\begin{array}{l}3 \\
9\end{array}$ & S & $\begin{array}{l}3 \\
7\end{array}$ & S & $\begin{array}{l}3 \\
4\end{array}$ & S & $\begin{array}{l}3 \\
7\end{array}$ & S & $\begin{array}{l}2 \\
9\end{array}$ & S & $\begin{array}{l}3 \\
0\end{array}$ & S & $\begin{array}{l}3 \\
5\end{array}$ & S & $\begin{array}{l}2 \\
7\end{array}$ & S & $\begin{array}{l}2 \\
8\end{array}$ & S & $\begin{array}{l}3 \\
0\end{array}$ & S & $\begin{array}{l}3 \\
7\end{array}$ \\
\hline $\begin{array}{l}\text { Tetracy } \\
\text { cline } \\
(30 \mu \mathrm{g})\end{array}$ & S & $\begin{array}{l}3 \\
0\end{array}$ & S & $\begin{array}{l}2 \\
4\end{array}$ & S & $\begin{array}{l}2 \\
7\end{array}$ & S & $\begin{array}{l}2 \\
4\end{array}$ & S & $\begin{array}{l}3 \\
0\end{array}$ & S & $\begin{array}{l}2 \\
9\end{array}$ & S & $\begin{array}{l}2 \\
5\end{array}$ & $\mathrm{R}$ & - & S & $\begin{array}{l}2 \\
7\end{array}$ & S & $\begin{array}{l}2 \\
3\end{array}$ & S & $\begin{array}{l}2 \\
2\end{array}$ & $\mathrm{R}$ & - & $\mathrm{R}$ & - & S & $\begin{array}{l}2 \\
3\end{array}$ & S & $\begin{array}{l}2 \\
9\end{array}$ \\
\hline $\begin{array}{l}\text { Methicy } \\
\text { cline } \\
(10 \mu \mathrm{g})\end{array}$ & S & $\begin{array}{l}2 \\
4\end{array}$ & S & $\begin{array}{l}2 \\
1\end{array}$ & S & $\begin{array}{l}2 \\
7\end{array}$ & $\mathrm{~S}$ & $\begin{array}{l}2 \\
1\end{array}$ & S & $\begin{array}{l}2 \\
4\end{array}$ & S & $\begin{array}{l}3 \\
7\end{array}$ & S & $\begin{array}{l}2 \\
6\end{array}$ & S & $\begin{array}{l}2 \\
2\end{array}$ & S & $\begin{array}{l}2 \\
7\end{array}$ & S & $\begin{array}{l}2 \\
8\end{array}$ & $\mathrm{R}$ & - & $\mathrm{R}$ & - & $\mathrm{R}$ & - & S & $\begin{array}{l}2 \\
8\end{array}$ & S & $\begin{array}{l}3 \\
7\end{array}$ \\
\hline $\begin{array}{l}\text { Ciproflo } \\
\text { xacin } \\
(30 \mu \mathrm{g})\end{array}$ & $S$ & $\begin{array}{l}4 \\
0\end{array}$ & S & $\begin{array}{l}3 \\
2\end{array}$ & S & $\begin{array}{l}3 \\
6\end{array}$ & S & $\begin{array}{l}3 \\
2\end{array}$ & S & $\begin{array}{l}4 \\
0\end{array}$ & S & $\begin{array}{l}3 \\
5\end{array}$ & S & $\begin{array}{l}2 \\
9\end{array}$ & S & $\begin{array}{l}3 \\
0\end{array}$ & S & $\begin{array}{l}3 \\
6\end{array}$ & S & $\begin{array}{l}3 \\
3\end{array}$ & $\mathrm{~S}$ & $\begin{array}{l}2 \\
9\end{array}$ & $\mathrm{~S}$ & $\begin{array}{l}2 \\
7\end{array}$ & $\mathrm{~S}$ & $\begin{array}{l}2 \\
6\end{array}$ & S & $\begin{array}{l}3 \\
3\end{array}$ & S & $\begin{array}{l}3 \\
5\end{array}$ \\
\hline $\begin{array}{l}\text { Chlorot } \\
\text { etracycl } \\
\text { ine }(30 \\
\mu \mathrm{g})\end{array}$ & $S$ & $\begin{array}{l}3 \\
7\end{array}$ & S & $\begin{array}{l}3 \\
4\end{array}$ & S & $\begin{array}{l}3 \\
8\end{array}$ & S & $\begin{array}{l}3 \\
4\end{array}$ & S & $\begin{array}{l}3 \\
7\end{array}$ & S & $\begin{array}{l}2 \\
6\end{array}$ & $\mathrm{R}$ & - & $\mathrm{R}$ & - & S & $\begin{array}{l}3 \\
8\end{array}$ & S & $\begin{array}{l}2 \\
4\end{array}$ & S & $\begin{array}{l}2 \\
9\end{array}$ & S & $\begin{array}{l}3 \\
7\end{array}$ & $\mathrm{R}$ & - & S & $\begin{array}{l}2 \\
4\end{array}$ & S & $\begin{array}{l}2 \\
6\end{array}$ \\
\hline $\begin{array}{l}\text { Kanamy } \\
\text { cin }(30 \\
\mu \mathrm{g})\end{array}$ & S & $\begin{array}{l}3 \\
2\end{array}$ & S & $\begin{array}{l}3 \\
1\end{array}$ & S & $\begin{array}{l}2 \\
1\end{array}$ & S & $\begin{array}{l}3 \\
1\end{array}$ & S & $\begin{array}{l}3 \\
2\end{array}$ & S & $\begin{array}{l}1 \\
6\end{array}$ & $\mathrm{R}$ & - & $\mathrm{R}$ & - & S & $\begin{array}{l}2 \\
1\end{array}$ & $\mathrm{R}$ & - & $\mathrm{R}$ & - & $\mathrm{R}$ & - & $\mathrm{R}$ & - & $\mathrm{R}$ & - & $S$ & $\begin{array}{l}1 \\
6\end{array}$ \\
\hline $\begin{array}{l}\text { Vancom } \\
\text { ycin }(30 \\
\mu \mathrm{g})\end{array}$ & S & $\begin{array}{l}1 \\
9\end{array}$ & S & $\begin{array}{l}2 \\
0\end{array}$ & S & 7 & S & $\begin{array}{l}2 \\
0\end{array}$ & S & $\begin{array}{l}1 \\
9\end{array}$ & S & $\begin{array}{l}1 \\
8\end{array}$ & S & $\begin{array}{l}1 \\
8\end{array}$ & S & $\begin{array}{l}2 \\
2\end{array}$ & S & 7 & S & $\begin{array}{l}1 \\
9\end{array}$ & S & $\begin{array}{l}1 \\
8\end{array}$ & S & $\begin{array}{l}1 \\
2\end{array}$ & $\mathrm{R}$ & - & S & $\begin{array}{l}1 \\
9\end{array}$ & S & $\begin{array}{l}1 \\
8\end{array}$ \\
\hline
\end{tabular}




\section{Conclusion}

From the present study it can be concluded that all the fifteen CDB isolated from mangrove soil of Mahanadi river delta could efficiently produce cellulase in the medium which could probably help taxonomists, enzymologists, and even some industrialists in their own researches. Use of these $\mathrm{CDB}$ as bio-inoculants can be incorporated to enhance organic matter decomposition in soil to increase soil fertility and to minimize the fertilizer application. They can also be apply to reduce environmental pollution and promotes sustainable agriculture.

\section{References}

[1] Gonzalez-Acosta, B., Bashan, Y., Hernandez-Sa-avedra, N. Y., Ascenaio, F. and Cruz-Aguero, G, "Seasonal Seawater Temperature as the Major Determinant for Populations of Culturable Bacteria in the Soils of an In-tact Mangrove in an Arid Region,” FEMS Microbiology Ecology, 55 (2). 311-321. Feb. 2006.

[2] Alongi, D. M, "The Role of Bacteria in Nutrient Recy-cling in Tropical Mangrove and Other Coastal Benthic Ecosystems,” Hydrobiologia, 285. 19-32. Jun. 1994.

[3] Holguin, G., Bashan, Y. and Vazavez, P, "The Role of Soil Microorganism in the Productivity, Conservation and Rehabilitation of Mangrove Ecosystem: An Overview,” Biology of Fertile Soils, 33 (4). 265-278. Feb. 2001.

[4] Rojas, A., Holguin, G., Glick, B. R. and Bashan, Y, "Syn-ergism between Phyllobacterium sp. (N2-Fixer) and Ba-cillus licheniformis (P-Solubilizer), both from a Semiarid Mangrove Rhizosphere" FEMS Microbiology Ecology, 35. 181-187. Apr. 2001.

[5] Cundell, A. M., Brown, M. S., Stanford, R. and Mitchell, R, "Microbial degradation of Rhizophora mangle leaves immersed in the sea,” Estuarine and coastal marine Science, 9. 281-286. Sept. 1979.

[6] Bobbie, J. and Leatherwood, J.M, "Derepressed synthesis of cellulose by Cellulomona,” Journal of Bacteriology, 128. 609-615. Nov. 1976.

[7] Nishida, Y., Suzuki, K.I., Kumagai, Y., Tanaka, H., Inoue, A. and Ojima, T, "Isolation and primary structure of a cellulase from the Japanese sea urchin” Strongylocentrotus nudus. Biochimie. 1-10. Aug. 2007.

[8] Nakari, S.T. and Penttila, M, "Production of Trichoderma ressei cellulases on glucose containing media" Applied and Environmental Microbiology, 61. 3650-36505. Oct. 1995.

[9] Kumakura, M, "Preparation of immobilized cellulase beads and their application to hydrolysisof cellulosic materials," Process Biochemistry, 32.555-559. Sept. 1997.
[10] Costa, R.B., Silva, M.V.A., Freitas, F.C., Leitao, V.S.F., Lacerda, P.S.B., Ferrara, M.A. and Bon, Eps, Mercado e perspectivas de Uso de Enzimas Industriaise Especiais no brasil. In Bon EPS, Ferrara MA, Corvo ML, Vermelho AB, Paiva CLA, Alencastro RB, Coelho RRR (eds) Enzimas em Biotechnologia, producao, Aplicac, oes e Mercados, $1^{\text {st }}$ edn. Reo de Janeiro, Interciencia, 2008. pp 463-488.

[11] Apun, K., Jong, B. C. and Salleh, M. A, "Screening and isolation of a cellulolytic and amylolytic Bacillus from sago pith waste," Journal of Genneral and Applied Microbilogy, 46. 263-267. Oct. 2000.

[12] Howard, R.L., Masoko, P. and Abotsi, E, "Enzyme activity of a Phanerochaete chryso-sporium cellobiohydrolase (CBHI. 1) expressed as a heterologous protein from Escherichia coli," African Journal of Biotechnology, 2 (9). 296-300. Sept. 2003.

[13] Ariffin, H. N., Abdullah, M.S., Umi Kalsom, Y., Shirai, and Hassan M.A, "Production and characterisation of cellulase by Bacilluspumilus EB3,” International Journal of Engeenering \& Technology, 3 (1). 47-53. Jun. 2006.

[14] Ray, A.K., Bairagi, A., Ghosh, K.S. and Sen S.K, "Optimization of fermentation conditions for cellulase production by Bacil l $\mathrm{u} s$ su btilis CY5 and Bacillus circulans TP3 isolated from fish gut," Acta Ichthyologica ET Piscatoria, 37 (1). 47-53. Jul. 2007.

[15] Mandels, M. and Weber, J: The production of cellulases. In: Cellulases and Their Appli-cations. Hajny, G.J. \& E.T. Reese (eds), American Chemical Society, Washington DC, 1969, 391414.

[16] Buchanan, R.E. and Gibbons, N.E, “Bergey's manual of determinative bacteriology. (Eighth edition), The Williams and Wilkins Co., Baltimore, 1974, pp. 747-842.

[17] Ramanathan, A.L., Singh, G., Majunder, J., Samal, A.C., Chahuan, R., Ranjan, R.K., Rajkumar, K. and Santra, S.C, "A study of microbial diversity and its interaction with nutrients in the sediment of Sunder ban mangrove," Indian Journal of Marine Science, 37. 159-165. June. 2008.

[18] Hatami, S., Alikhani, H.A., Besharati, H. N., Salehrastin, M., Afrousheh, and Yazdani Jahromi, "Investigation on aerobic cellulolytic bacteria in some of north forest and farming soils," Biotechnology and Bioengineering Symposium, 5. 193-219. 2008.

[19] Lu, W.J., Wang, H.T., Yang, S.J., Wang, Z.C. and Nie, Y.F, "Isolation and characterisation of mesophilic cellulose degrading bacteria from flower stalks-vegetable waste co-composting system,” Journal of General Applied Microbiology, 51. 353-360. Dec. 2005.

[20] Gupta, P., Samant, K. and Sahu, A, "Isolation of cellulosedegrading bacteria and determination of their cellulolytic potential,” International Journal of Microbiology, Oct. 2012.

[21] Tabao, N.S.C. and Monsalud, R.G, "Characterization and identification of high cellulose producing bacterial strains from philippine mangroves,” Philipine journal of Systemetic Biology, 4. 13-20. June. 2010.

[22] Mukesh Kumar, D.J., Poovai P.D., Puneeth Kumar, C.L., Sushma Saroja, Y., Manimaran, A. and Kalaichelvan, P.T, "Optimization of Bacillus cereus MRK1 cellulase production and its Biostoning activity” Der Pharmacia Lettrer, 4 (3). 881-888. 2012.

[23] Thatoi, H.N., Behera, B.C., Dangar, T.K. and Mishra, R.R, "Microbial biodiversity in mangrove soil of Bhitarakanika, Odisha, India,” International Journal of Environmental Biology, 2 (2). 5058. Apr. 2012. 